Poster \#7

\section{Phylogenetic Relationships of Algal Symbionts of Azolla and Free-Living Cyanobacterial Cultures Based on DNA Amplification}

\author{
V.B. Konde \& S. Kannaiyan
}

Centre for Plant Molecular Biology, Tamil Nadu Agricultural University, Coimbatore-641 003, Tamil Nadu, India

Azolla is a free floating water fern which fixes atmospheric nitrogen in association with nitrogen-fixing cyanobacterium Anabaena azollae. Random amplified polymorphic DNA technique was used to generate fingerprinting in symbiotic Anabaena azollae isolates viz., $A$. azollae-VBK-SK-AM, $A$. azollae-VBK-SK-AF, $A$. azollae-VBK-SK-TNAU and $A$. azollae-VBK-SK-RP as well as free-living cyanobacterial cultures viz., Anabaena variabilis, Nostoc muscorum (DOH), Westiellopsis sp. (Kew-SK) and Oscillatoria sp. A total of 15 random primers were used and the bands were scored as present or absent for each cyanobacterial cultures. The variation among cyanobacterial cultures was quantified using Nei's genetic distances. A dendrogram summarizing phenetic relationship among the cyanobacterial cultures was generated using the unweighted pair-group method with arithmetic mean. The statistical analysis was performed using "RAPDistance" 1.04, a computer program developed by John Armstrong (Research School of Biological Sciences, Institute of Advanced Studies, A.N.U., Australia). Three distinct groups were identified: group 1 contains four symbiotic $A$. azollae isolates, group 2 contains the free-living cyanobacterial cultures viz., A. variabilis, Nostoc muscorum (DOH) and Oscillatoria $s p$. whereas, Westiellopsis sp. (Kew-SK) was placed in the third group. The analysis demonstrated that the major cyanobacterial symbiont Anabaena azollae can be easily distinguished from the free-living cyanobacterial cultures.
Poster \#8

\section{Cationic Peptide Expression in Transgenic Potato Confers Broad-Spectrum Resistance to Phytopathogens}

\author{
Milan Osusky, Lubica Osuska, William W. Kay \\ \& Santosh Misra
} Department of Biochemistry and Microbiology, University of Victoria,
Victoria, B.C. V8W 3P6, Canada

Broad-spectrum resistance to pathogens and spoilage organisms is a major goal of plant molecular biologists. To provide broad-spectrum resistance to bacterial and fungal phytopathogens, a synthetic gene encoding a modified chimeric, cecropin-melittin cationic peptide (MsrA1), with broad-spectrum antimicrobial activity, was introduced into two potato (Solanum tuberosum L.) cultivars, Desiree and Russet Burbank, under the control of the duplicated-enhancer CaMV $35 \mathrm{~S}$ promoter. The 34 -amino acid peptide (MsrA1) comprises, 8 amino acids from cecropin $\mathrm{A}$ and 16 amino acids from melittin but with a modified hexapeptide $\mathrm{N}$-terminus to dampen cytotoxicity. Incorporation was confirmed by PCR and DNA sequencing and expression by RT-PCR. Unusually stringent cocultivation bioassays of the resulting transgenic plants with phytopathogenic bacterial or fungal pathogens indicated powerful resistance to these phytopathogens. The morphology and yield of transgenic Desiree tubers were comparable to that of non-transformed plants. Tubers were shown to retain their resistance to bacterial infection after several months of storage. In Russet Burbank, expression of $m s r A l$ also caused a lesionmimic phenotype displayed during leaf and tuber development. Thus the expression of $m s r A l$ confers a powerful, broadspectrum, phytopathogen-resistant phenotype to transgenic potato plants and tubers, and provides a novel and highly flexible strategy for the creation of new generations of disease and spoilage resistant plants. 